SUPPORTING INFORMATION

\title{
Development of Omniphobic Desalination Membranes Using a Charged Electrospun Nanofiber Scaffold
}

ACS Applied Materials and Interfaces

\author{
Jongho Lee,,$^{\dagger}$ Chanhee Boo, ${ }^{\dagger}$ Won-Hee Ryu, ${ }^{\dagger, t}$ André D. Taylor ${ }^{\dagger}$ \\ and Menachem Elimelech ${ }^{*},, \xi$
}

$\dagger$ Department of Chemical and Environmental Engineering, Yale University, New Haven, Connecticut 06520-8286

*Department of Chemical \& Biological Engineering, Sookmyung Women's University, 100 Cheongpa-ro 47-gil, Yongsan-gu, Seoul 04310, Republic of Korea

${ }^{\S}$ Nanosystems Engineering Research Center for Nanotechnology-Enabled Water Treatment (NEWT), Yale University

* Corresponding author: Menachem Elimelech

Email: menachem.elimelech@yale.edu, Phone: (203) 432-2789 


\section{S1. Measurement of Effective Pore Size Distribution Using Capillary Flow Porometry}

The effective pore size distributions of the PVDF-HFP and PVDF-HFP/BTEAC membranes were obtained from a custom-built capillary flow porometer using the commonly used wet/dry flow method. ${ }^{1-2}$ The porometer comprises a stainless steel filter holder (KS 13, Advantec MFS), a pressure gauge (Duro United Instrument), and a gas flow meter (MPC 05, Parker Hannifin Corp.). The cut membrane coupon with a diameter of $\sim 1.2 \mathrm{~cm}$ was mounted on the filter holder, with a perforated stainless steel screen support (50\% open). Teflon O-rings were used on each side of the membrane for complete sealing and the holder was tightly screwed. Dry nitrogen (Airgas) was used as a gas source and pressure at the inlet of the holder was controlled by a pressure regulator (Airgas). The flow rate of nitrogen through the membrane was measured by a flow meter.

For 'dry' runs, the membrane coupon was mounted on the filter holder and nitrogen was introduced into the holder with a gauge pressure increasing from 0 to $130 \mathrm{kPa}$ with increments of $2 \mathrm{kPa}$. For 'wet' runs, the membrane coupon was first wetted by fluoroethane (surface tension of $\sim 16 \mathrm{mN} / \mathrm{m}$ ) and then mounted on the holder. Using the same pressure range as in the 'dry' runs, the nitrogen gas flow rates were measured by a flow meter. The ratio of gas flow rates obtained from the 'wet' run to those from the 'dry' run enables us to obtain the fraction of pores with an effective diameter at the designated pressure. ${ }^{1-2}$ Briefly, the effective diameter, $d$, of pores (previously wetted by fluoroethane) that displace the wetting liquid (i.e., fluoroethane) with nitrogen at each pressure was estimated from the Laplace equation, i.e., $d=4 \sigma \cos \theta / \Delta p$, where $\sigma$ is the surface tension of the wetting liquid (i.e., $\sigma=16 \mathrm{mN} / \mathrm{m}$ ), $\theta$ is the contact angle (assumed to be zero), and $\Delta p$ is the transmembrane pressure (i.e., nitrogen gas pressure). Based on the incremental ratio of flow rates between the dry and wet runs at each pressure, the number of pores with the corresponding effective diameter $(d)$ and their fraction to the total number of pores were obtained (Figure S1). The average effective diameter of the pores of PVDF-HFP/BTEAC was estimated to be $0.42 \pm 0.09 \mu \mathrm{m}$, significantly smaller than $2.23 \pm 0.26 \mu \mathrm{m}$ of PVDF-HFP. 

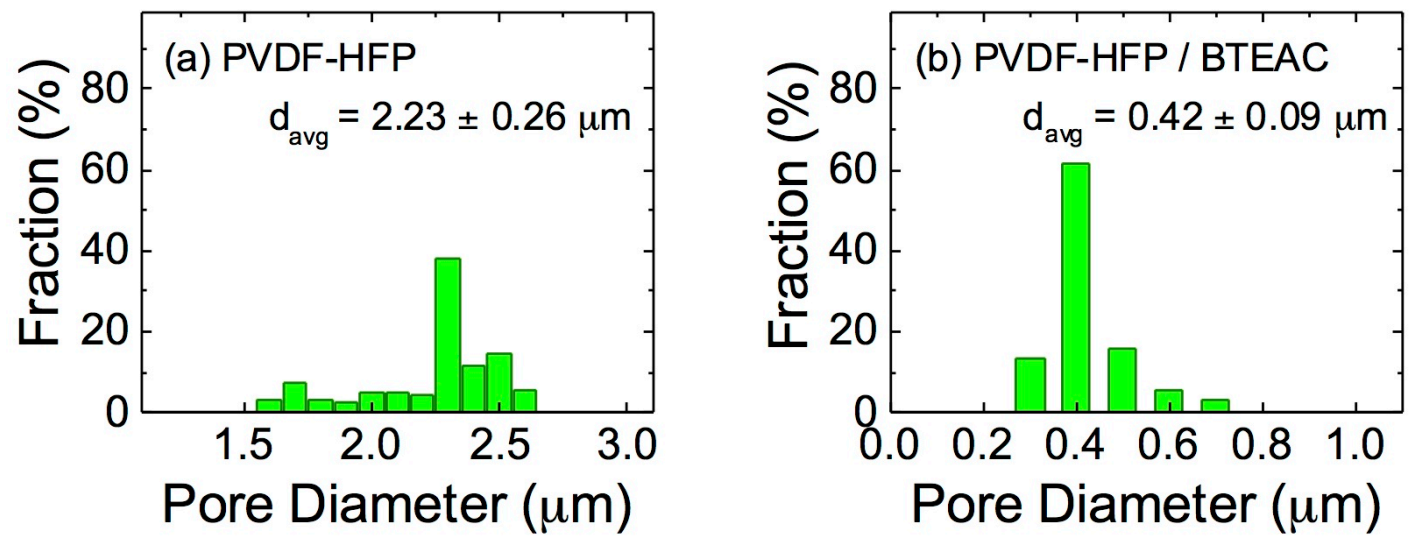

Figure S1. Distribution of effective pore sizes of (a) PVDF-HFP and (b) PVDF-HFP / BTEAC membranes.

\section{S2. Robustness of Bonding between SiNPs and Nanofibers}

The robustness of bonding between the SiNPs and the nanofibers was tested by exposing the SiNPs-PVDF-HFP/BTEAC membrane (after dip-coating) to physical stress. The membrane was immersed in an acetate buffer at $\mathrm{pH} 4$ and sonicated for 3 min. As shown in Figure S2(c, d), the SiNPs remained firmly attached to the nanofibers, demonstrating irreversible binding once they are grafted via electrostatic attraction. In addition, the fluorination step may further enhance the binding stability of the SiNPs due to the chemically crosslinked fluorosilane layers "wrapping" the SiNPs. SEM images of the membrane after $8 \mathrm{~h}$ of MD experiments for water separation again revealed the same intact binding of SiNPs on the nanofibers (Figure S2(e, f)). 

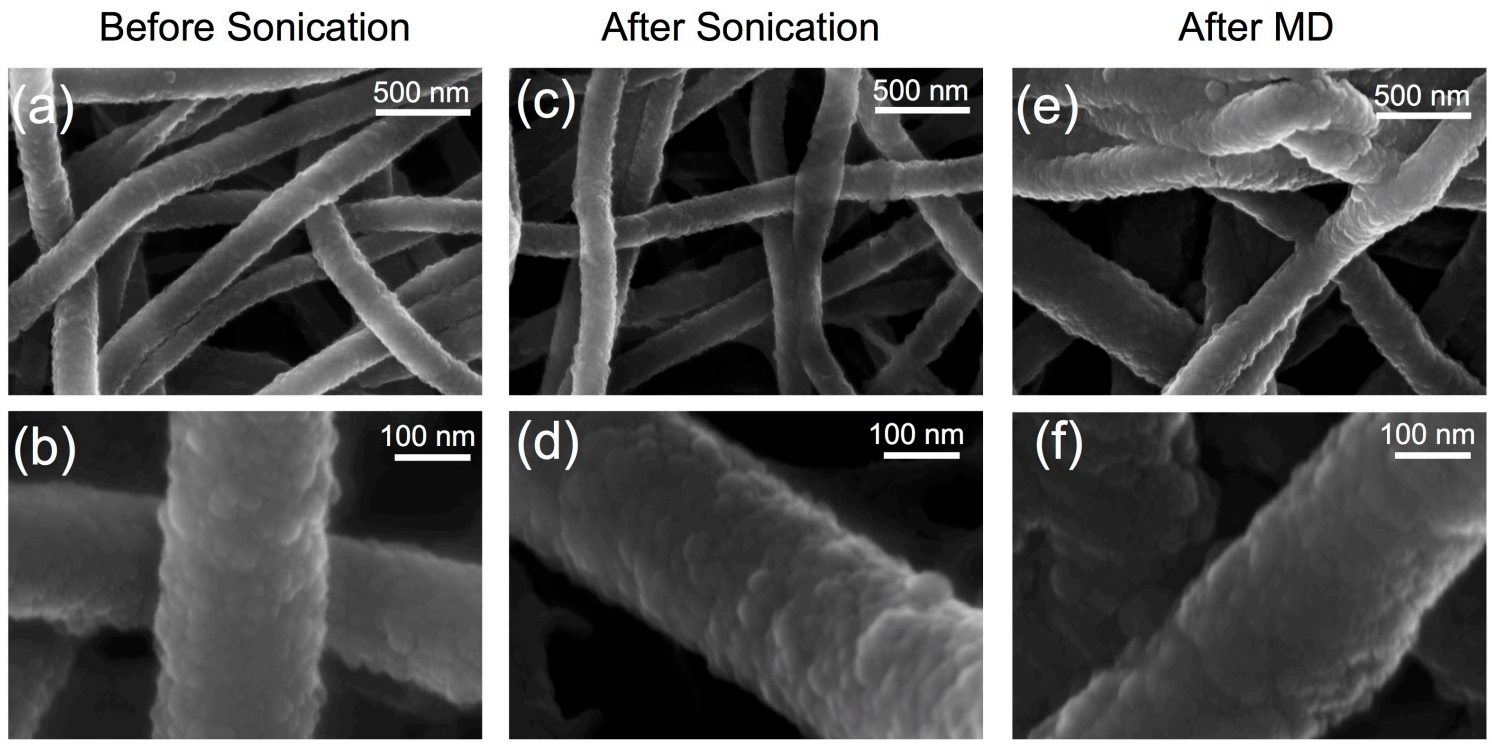

Figure S2. Robust bonding between SiNPs and nanofibers of PVDF-HFP / BTEAC membranes. SEM images of the nanofibers before $(a, b)$ and after (c, d) 3 min sonication in sodium acetate buffer solution at $\mathrm{pH} 4$ with an ionic strength of approximately $1 \mathrm{mM}$. (e) and (f) SEM images of the nanofibers after $8 \mathrm{~h}$ of direct contact MD experiment. (a), (c), (e) and (b), (d), (f) show low and high magnification images, respectively.

\section{S3. Effects of Dip-coating Conditions on SiNPs Grafting}

In order to investigate the effect of dip-coating conditions on SiNP grafting quality, we performed dip-coating of the PVDF-HFP/BTEAC membrane in SiNP suspensions with three different concentrations (i.e., $0.005 \mathrm{wt} \%, 0.05 \mathrm{wt} \%$, and $0.5 \mathrm{wt} \%$ ) prepared in an acetate buffer at $\mathrm{pH} 4$ for 3 min. All dip-coated membrane samples exhibited dense coverage of nanofibers with SiNPs, indicating the insignificance of the tested SiNP concentrations on SiNP grafting density (Figure S3). In addition, the high SiNP grafting density obtained for the short time duration (3 $\mathrm{min})$, the same extent as the $1 \mathrm{~h}$ duration (Figure 2 and S2), indicates that electrostatic force between the SiNPs and the nanofibers is quite effective after the membrane is immersed in the solution. 

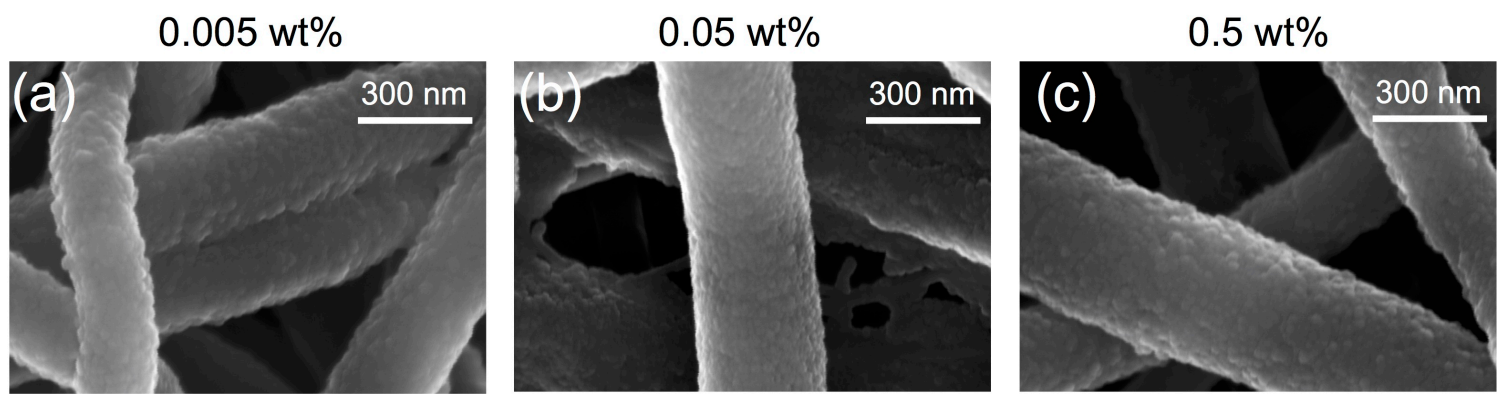

Figure S3. Effect of different SiNP concentrations on grafting density. SEM images of the nanofibers of PVDF-HFP/BTEAC membranes after 3 min dip-coating in $0.005 \mathrm{wt} \%, 0.05 \mathrm{wt} \%$, and $0.5 \mathrm{wt} \% \mathrm{SiNP}$ concentration prepared in an acetate buffer at $\mathrm{pH} 4$ with an ionic strength of approximately $1 \mathrm{mM}$.

\section{S4. Estimation of Mass Fraction of Grafted SiNPs}

Based on the change in total mass obtained from TGA, we estimated mass fraction of SiNPs grafted on PVDF-HFP and PVDF-HFP/BTEAC nanofiber mats after dip-coating in SiNP suspension (i.e., SiNPs-PVDF-HFP and SiNPs-PVDF-HFP/BTEAC mats, respectively). For the SiNPs-PVDF-HFP/BTEAC mat, the fraction of total mass relative to the initial total mass at any temperature during TGA is defined as:

$$
a \equiv \frac{m_{p}+m_{s}+m_{N P}+R}{m_{p, \max }+m_{s, \max }+m_{N P}+R}=\frac{m_{p}+m_{s}+m_{N P}+q m_{p, \max }}{m_{p, \max }+m_{s, \max }+m_{N P}+q m_{p, \max }}
$$

where $m_{p, \max }$ and $m_{s, \max }$ are the maximum masses of PVDF-HFP and BTEAC (i.e., initial masses), respectively. $m_{p}$ and $m_{s}$ are masses of PVDF-HFP and BTEAC in the mat, respectively, at any given temperature. $m_{N P}$ indicates mass of SiNPs grafted on the nanofibers. $R$ is residual mass accounting for any impurities that were not decomposed above $700^{\circ} \mathrm{C}$, and $q \equiv R / m_{p \text {, max }}$.

If we define another parameter $d$ as a fraction of $m_{s}$ to the total initial mass, $m_{s}$ can be expressed as:

$m_{s}=d\left[(1+q) m_{p, \text { max }}+m_{s, \text { max }}+m_{N P}\right]$

The following relation also holds:

$m_{s, \max }=d_{\max }\left[(1+q) m_{p, \max }+m_{s, \max }+m_{N P}\right]$

where $d_{\max }$ is the largest (i.e., initial) mass fraction of BTEAC. Rearranging the above equations leads to: 


$$
a=\frac{\left(1-d_{\max }\right)\left(m_{p}+m_{N P}+q m_{p, \max }\right)}{(1+q) m_{p, \text { max }}+m_{N P}}+d
$$

After complete decomposition of PVDF-HFP and BTEAC, $a_{\min }$, which is the minimum value of $a$, can be given as:

$$
a_{\min }=\frac{\left(1-d_{\max }\right)\left(m_{N P}+q m_{p, \text { max }}\right)}{(1+q) m_{p, \text { max }}+m_{N P}}
$$

with $m_{p}=0$ and $d=0$. Another variable $c$, the mass fraction of SiNPs to the initial mass of PVDF-HFP, can then be expressed as:

$$
c \equiv \frac{m_{N P}}{m_{p, \max }}=\frac{(1+q) a_{\min }-q\left(1-d_{\max }\right)}{1-d_{\max }-a_{\min }}
$$

where $a_{\min }$ and $d_{\max }$ were directly obtained from the minimum value of $a$, (i.e., $a_{\min }=0.043$ ), and from the observed mass fraction of BTEAC during decomposition, (i.e., $d_{\max }=0.0056$ ), respectively (Figure 4d). Note that although the mass fraction of BTEAC relative to PVDF-HFP in the PVDF-HFP/BTEAC mat was initially 0.10 (Figure 4c), a significant portion of BTEAC was dissolved in the SiNP suspension during the dip-coating (see Section 3.2 in the manuscript).

The values of $q$ were calculated from the minimum values of $a$ measured by TGA for nanofiber mats without SiNP coating (i.e., PVDF-HFP and PVDF-HFP/BTEAC mats). By substituting $m_{\mathrm{NP}}=0, d_{\max }=0$ for PVDF-HFP mat and $d_{\max }=0.1$ for PVDF-HFP/BTEAC mat in

equation S5, the average value of $q$ was obtained as $0.0245 \pm 0.0080$. With $a_{\min }=0.043$ and $d_{\max }$ $=0.1$ for the SiNPs-PVDF-HFP/BTEAC mat, $m_{\mathrm{NP}} / m_{p, \max }$ was determined to be $2.01 \%$ (Figure $4 \mathrm{~d}$, right inset). Through the same procedure, $m_{\mathrm{NP}} / m_{p, \max }$ for the SiNPs-PVDF-HFP mat without BTEAC was obtained as $0.055 \%$, with $a_{\min }=0.0246$ and $d_{\max }=0$ (Figure $4 \mathrm{~d}$, right inset). This analysis clearly shows that substantially larger amount of SiNPs were grafted on the PVDFHFP/BTEAC nanofiber mat than that without BTEAC, due to electrostatic attraction.

\section{S5. Long-term Performance of Omniphobic Membranes for Membrane Distillation}

The robustness and long-term stability of the omniphobic membrane were evaluated in a separate MD experiment. The SiNPs-PVDF-HFP/BTEAC membrane (i.e., SiNPs grafted membrane) was first subjected to bath sonication for $3 \mathrm{~min}$ and then silanized to achieve surface omniphobicity. A dense grafting of SiNPs on the nanofibers was again observed as previously shown in Figure S2. We performed $\mathrm{MD}$ experiments using $1 \mathrm{M} \mathrm{NaCl}$ at $60{ }^{\circ} \mathrm{C}$ and deionized water at $20{ }^{\circ} \mathrm{C}$ as feed 
and permeate solutions, respectively. Sodium dodecyl sulfate (SDS) was sequentially dosed into the feed solution at intervals of $4 \mathrm{~h}$, leading to concentrations of $0.1 \mathrm{mM}, 0.2 \mathrm{mM}$, and $0.3 \mathrm{mM}$, which resulted in feed surface tensions of $\sim 41 \mathrm{mN} / \mathrm{m}, \sim 33 \mathrm{mN} / \mathrm{m}$, and $\sim 31 \mathrm{mN} / \mathrm{m}$, respectively. Throughout the entire operation for $20 \mathrm{~h}$, the omniphobic membrane exhibited complete salt rejection as well as stable water flux, which demonstrates an excellent long-term stability of our omniphobic MD membrane for desalination.

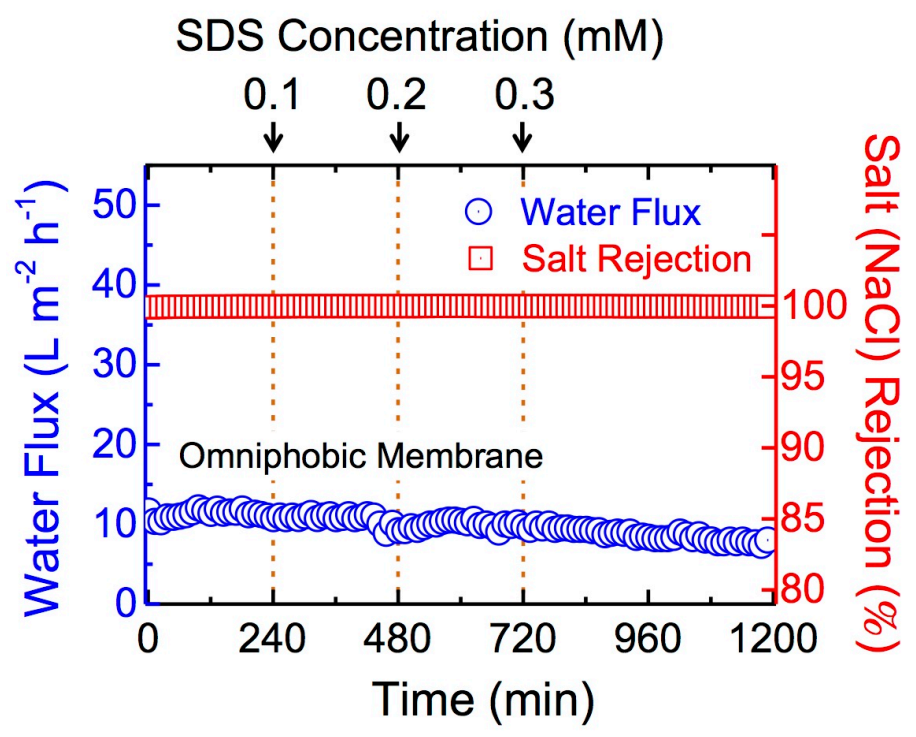

Figure S4. Long-term performance of the omniphobic membrane for desalination in a direct contact membrane distillation (MD) experiment. Time traces of water vapor flux across the membranes and salt rejection for the omniphobic membrane, based on sequential doses of sodium dodecyl sulfate (SDS) with 4 $\mathrm{h}$ intervals. 